\title{
Distance between Quantum States and Gauge-Gravity Duality
}

\section{$\operatorname{AUTHOR}(\mathrm{S})$ :}

Miyaji, Masamichi; Numasawa, Tokiro; Shiba, Noburo; Takayanagi, Tadashi; Watanabe, Kento

\section{CITATION:}

Miyaji, Masamichi ...[et al]. Distance between Quantum States and Gauge-Gravity Duality. Physical Review Letters 2015, 115(26): 261602.

\section{ISSUE DATE:}

2015-12-31

URL:

http://hdl.handle.net/2433/252426

\section{RIGHT:}

(c) 2015 American Physical Society; 許諾条件に基づいて掲載しています $\circ$ 


\title{
Distance between Quantum States and Gauge-Gravity Duality
}

\author{
Masamichi Miyaji, ${ }^{1}$ Tokiro Numasawa, ${ }^{1}$ Noburo Shiba, ${ }^{1}$ Tadashi Takayanagi, ${ }^{1,2}$ and Kento Watanabe ${ }^{1}$ \\ ${ }^{1}$ Yukawa Institute for Theoretical Physics, Kyoto University, Kitashirakawa Oiwakecho, Sakyo-ku, Kyoto 606-8502, Japan \\ ${ }^{2}$ Kavli Institute for the Physics and Mathematics of the Universe, University of Tokyo, Kashiwa, Chiba 277-8582, Japan
}

(Received 3 August 2015; revised manuscript received 5 October 2015; published 22 December 2015)

\begin{abstract}
We study a quantum information metric (or fidelity susceptibility) in conformal field theories with respect to a small perturbation by a primary operator. We argue that its gravity dual is approximately given by a volume of maximal time slice in an anti-de Sitter spacetime when the perturbation is exactly marginal. We confirm our claim in several examples.
\end{abstract}

DOI: 10.1103/PhysRevLett.115.261602

PACS numbers: 11.25.Hf, 11.25.Tq, 04.60.Cf

The microscopic understanding of black hole entropy in string theory by Strominger and Vafa [1] implies that quantum information plays a crucial role in understanding gravitational aspects of string theory. Indeed, quantum information theoretic considerations have provided various useful viewpoints in studies of AdS/CFT [2] or more generally holography [3]. Especially, the idea of quantum entanglement has turned out to be crucially involved in geometries of holographic spacetimes, as is typical in the nontrivial topology of eternal black holes [4]. To quantify quantum entanglement we can study the holographic entanglement entropy [5], which is given by the area of codimension two extremal surfaces. In AdS/CFT, this area is equal to the entanglement entropy in conformal field theories (CFTs).

It is natural to wonder if there might be some other information theoretic quantities that are useful to develop studies of holography. As pointed out by Susskind in [6] (see also [7]), it is also intriguing to find a quantity in CFTs which is dual to a volume of a codimension one time slice in anti-de Sitter (AdS). The time slice can connect two boundaries dual to the thermofield doubled CFTs, through the Einstein-Rosen bridge (see Fig. 1). In [6], it is conjectured that this quantity is related to a measure of complexity.

The main purpose of this Letter is to point out a quantum information theoretic quantity that is related to the volume of a time slice. This quantity is called quantum information metric or Bures metric (see e.g.[8]), which we will simply call the information metric. Here we mainly consider the information metric for pure states, though it can be defined for mixed states. Consider one parameter family of quantum states $|\Psi(\lambda)\rangle$ and perturb $\lambda$ infinitesimally as $\lambda \rightarrow \lambda+\delta \lambda$. Then $G_{\lambda \lambda}$ is simply defined from the inner product between them as follows:

$$
|\langle\Psi(\lambda) \mid \Psi(\lambda+\delta \lambda)\rangle|=1-G_{\lambda \lambda}(\delta \lambda)^{2}+O\left((\delta \lambda)^{3}\right) .
$$

This metric measures the distance between two infinitesimally different quantum states. Since the left-hand side of (1) is called the fidelity, $G_{\lambda \lambda}$ is also called the fidelity susceptibility. This quantity gets divergent at quantum critical points and thus can be used as an order parameter of quantum phase transitions (see e.g. the review [9]).

We will argue that $G_{\lambda \lambda}$ when a $d+1$ dimensional CFT is deformed by an exactly marginal perturbation, parametrized by $\lambda$, is holographically estimated by

$$
G_{\lambda \lambda}=n_{d} \frac{\operatorname{Vol}\left(\Sigma_{\max }\right)}{R^{d+1}},
$$

where $n_{d}$ is an $O(1)$ constant and $R$ is the AdS radius. The $d+1$ dimensional spacelike surface $\Sigma_{\max }$ is the time slice with the maximal volume in the AdS that ends on the time slice at the AdS boundary(boundaries). See also [10] for other holographic interpretations of information metric.

Now we introduce the information metric for quantum states in CFTs on $R^{d+1}$, whose Euclidean time and space coordinates are denoted by $\tau$ and $x$. We consider the inner product $\left\langle\Omega_{1} \mid \Omega_{2}\right\rangle$ between two states $\left|\Omega_{1}\right\rangle$ and $\left|\Omega_{2}\right\rangle . \quad\left|\Omega_{i}\right\rangle(i=1,2)$ are ground states for the two Hamiltonians $H_{i}(i=1,2)$. We define their Euclidean Lagrangians by $\mathcal{L}_{i}(i=1,2)$ and their partition functions by $Z_{i}(i=1,2)$. The inner product is described by the path integral:

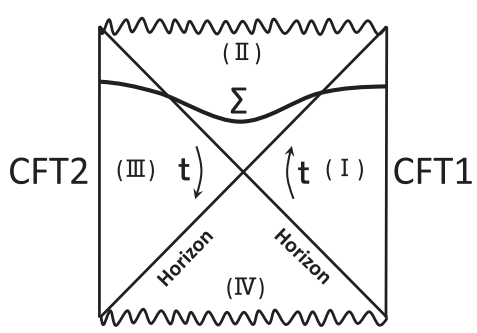

FIG. 1. A time slice in the Penrose diagram of eternal AdS black hole which connects the two boundaries dual to the thermofield doubled CFTs. 


$$
\begin{aligned}
\left\langle\Omega_{2} \mid \Omega_{1}\right\rangle= & \left(Z_{1} Z_{2}\right)^{-1 / 2} \int D \phi \exp \left[-\int d^{d} x\left(\int_{-\infty}^{0} d \tau \mathcal{L}_{1}\right.\right. \\
& \left.\left.+\int_{0}^{\infty} d \tau \mathcal{L}_{2}\right)\right] .
\end{aligned}
$$

Assume the difference $\mathcal{L}_{2}-\mathcal{L}_{1}$ is infinitesimally small and is written by using the primary operator $O(\tau, x)$ as

$$
\mathcal{L}_{2}-\mathcal{L}_{1} \equiv \delta \mathcal{L}=\delta \lambda O(\tau, x) .
$$

Next, we rewrite (3) by using the expectation value $\langle\cdots\rangle$ in the vacuum state $\left|\Omega_{1}\right\rangle$ :

$$
\left\langle\tilde{\Omega}_{2}(\epsilon) \mid \Omega_{1}\right\rangle=\frac{\left\langle\exp \left[-\int_{\epsilon}^{\infty} d \tau \int d^{d} x \delta \mathcal{L}\right]\right\rangle}{\left(\left\langle\exp \left[-\left(\int_{-\infty}^{-\epsilon}+\int_{\epsilon}^{\infty}\right) d \tau \int d^{d} x \delta \mathcal{L}\right]\right\rangle\right)^{1 / 2}},
$$

where $\delta \mathcal{L} \equiv \mathcal{L}_{2}-\mathcal{L}_{1}$. Here we introduced the UV regularization $\epsilon$ by replacing the ground state $\left|\Omega_{2}\right\rangle$ with

$$
\left|\tilde{\Omega}_{2}(\epsilon)\right\rangle \equiv \frac{e^{-\epsilon H_{1}}\left|\Omega_{2}\right\rangle}{\left(\left\langle\Omega_{2}\left|e^{-2 \epsilon H_{1}}\right| \Omega_{2}\right\rangle\right)^{1 / 2}} .
$$

By performing perturbative expansions of (5) up to quadratic terms, we obtain

$$
\begin{aligned}
1- & \left\langle\tilde{\Omega}_{2}(\epsilon) \mid \Omega_{1}\right\rangle \\
& =\frac{1}{2} \int_{\epsilon}^{\infty} d \tau \int_{-\infty}^{-\epsilon} d \tau^{\prime} \int d^{d} x \int d^{d} x^{\prime}\left\langle\delta \mathcal{L}(\tau, x) \delta \mathcal{L}\left(\tau^{\prime}, x^{\prime}\right)\right\rangle,
\end{aligned}
$$

where we assumed the time reversal symmetry relation $\left\langle\delta \mathcal{L}(-\tau, x) \delta \mathcal{L}\left(-\tau^{\prime}, x^{\prime}\right)\right\rangle=\left\langle\delta \mathcal{L}(\tau, x) \delta \mathcal{L}\left(\tau^{\prime}, x^{\prime}\right)\right\rangle$.

In this way, the information metric with respect to the $\lambda$ perturbation (4) is computed as

$$
G_{\lambda \lambda}=\frac{1}{2} \int_{\epsilon}^{\infty} d \tau \int_{-\infty}^{-\epsilon} d \tau^{\prime} \int d^{d} x \int d^{d} x^{\prime}\left\langle O(\tau, x) O\left(\tau^{\prime}, x^{\prime}\right)\right\rangle .
$$

Note that up to now, our argument can be applied to any local operator $O$ in any quantum field theory. However, for simplicity, we would like to focus on the case where the spin of the primary field $O$ is zero and its total conformal dimension is $\Delta$ in this Letter.

The (normalized) two-point function of the primary field takes the universal form

$$
\left\langle O(\tau, x) O\left(\tau^{\prime}, x^{\prime}\right)\right\rangle=\frac{1}{\left[\left(\tau-\tau^{\prime}\right)^{2}+\left(x-x^{\prime}\right)^{2}\right]^{\Delta}} .
$$

By plugging (8) into (7), when $d+2-2 \Delta<0$ we obtain

$$
G_{\lambda \lambda}=N_{d} V_{d} \epsilon^{d+2-2 \Delta},
$$

where we define $N_{d}=\left[2^{d-2 \Delta} \pi^{d / 2} \Gamma(\Delta-d / 2-1) /(2 \Delta-\right.$ $d-1) \Gamma(\Delta)]$ and $V_{d}$ is the infinite volume of $R^{d}$. In particular, for a marginal perturbation we have $d+2-$ $2 \Delta=-d$. On the other hand, when $d+2-2 \Delta \geq 0$, there exists an infrared (IR) divergence and we need an IR cutoff $L$ for both the $\tau$ and $x$ integral. This leads to $G_{\lambda \lambda} \propto$ $V_{d} L^{d+2-2 \Delta}$ (agreeing with [9]), where for $d+2-2 \Delta=0$ we regard $L^{0}$ as $\log (L / \epsilon)$.

Now we would like to turn to holographic calculations. For this we focus on the case where the perturbation (4) is exactly marginal $\Delta=d+1$. This greatly simplifies the computation in the gravity dual. This is because gravity backgrounds dual to both of $\left|\Omega_{i}\right\rangle(i=1,2)$ are the pure $\mathrm{AdS}_{d+2}$ with the same radius $R$. Such a gravity dual which interpolates two AdS spaces is called a Janus solution [11]. The massless bulk scalar field dual to the exactly marginal operator $O$ is denoted by $\phi$.

Let us first study the $\mathrm{AdS}_{3}$ Janus solution introduced in [12]. This setup is defined by the action

$$
S=-\frac{1}{16 \pi G_{N}} \int d x^{3} \sqrt{g}\left(\mathcal{R}-g^{a b} \partial_{a} \phi \partial_{b} \phi+\frac{2}{R^{2}}\right) .
$$

The Janus solution is given by the metric

$$
\begin{aligned}
d s^{2} & =R^{2}\left(d y^{2}+f(y) d s_{\mathrm{AdS} 2}^{2}\right), \\
f(y) & =\frac{1}{2}\left[1+\sqrt{1-2 \gamma^{2}} \cosh (2 y)\right],
\end{aligned}
$$

and the dilaton

$$
\phi(y)=\gamma \int_{-\infty}^{y} \frac{d y}{f(y)}+\phi_{1}
$$

where $\gamma[\leq(1 / \sqrt{2})]$ is the parameter of Janus deformation. The metric of $\mathrm{AdS}_{2}$ slice is given by $d s_{\text {AdS2 }}^{2}=\left(d z^{2}+d x^{2}\right) / z^{2} . \quad \phi_{1}=\phi(-\infty)$ is dual to the coupling constant of the exactly marginal deformation for the ground state $\left|\Omega_{1}\right\rangle$. On the other hand, the value $\phi_{2}=\phi(\infty)$ for the other ground state $\left|\Omega_{2}\right\rangle$ is obtained by performing the integral in (12) as $\phi_{2}-\phi_{1}=$ $\sqrt{2} \arctan \left[\left(1-\sqrt{1-2 \gamma^{2}}\right) / \sqrt{2} \gamma\right] \simeq \gamma$ when $\gamma \ll 1$.

By matching the asymptotic behavior of the metric (11) at the infinity $|y|=y_{\infty}(\rightarrow \infty)$ with that of an undeformed metric $(\gamma=0)$

$$
d s_{\text {pure }}^{2}=R^{2}\left[d \hat{y}^{2}+\frac{1}{2}(1+\cosh (2 \hat{y})) d s_{\text {AdS2 }}^{2}\right],
$$

we find the following condition: 


$$
\sqrt{1-2 \gamma^{2}} e^{2 y_{\infty}}=e^{2 \hat{y}_{\infty}}
$$

The on-shell action of (10) is evaluated by

$$
\begin{aligned}
S(\gamma) & =\frac{R}{4 \pi G_{N}} V_{\mathrm{AdS}_{2}} \int_{-y_{\infty}}^{y_{\infty}} d y\left\{\frac{1}{2}\left[1+\sqrt{1-2 \gamma^{2}} \cosh (2 y)\right]\right\} \\
& =\frac{R}{4 \pi G_{N}} V_{\mathrm{AdS}_{2}}\left\{y_{\infty}+\frac{1}{2} \sqrt{1-2 \gamma^{2}} \sinh \left(2 y_{\infty}\right)\right\},
\end{aligned}
$$

where $V_{\mathrm{AdS}_{2}}=\int d x \int_{\epsilon}^{\infty}\left(d z / z^{2}\right)=V_{1} / \epsilon$ is the volume of $\mathrm{AdS}_{2}$ with a unit radius.

By using the condition (14) at the infinity

$$
\begin{aligned}
S(\gamma)-S(0) & =\frac{R}{4 \pi G_{N}} V_{\mathrm{AdS}_{2}}\left(y_{\infty}-\hat{y}_{\infty}\right) \\
& =\frac{R V_{1}}{16 \pi G_{N} \epsilon} \log \left(\frac{1}{1-2 \gamma^{2}}\right)>0 .
\end{aligned}
$$

For small $\gamma^{2}$, we finally find

$$
\left|\left\langle\Omega_{2} \mid \Omega_{1}\right\rangle\right|=e^{-S(\gamma)-S(0)} \simeq 1-\frac{R V_{1}}{8 \pi G_{N} \epsilon} \gamma^{2} .
$$

Therefore the information metric is estimated as follows:

$$
G_{\gamma \gamma}=\frac{c V_{1}}{12 \pi \epsilon},
$$

where we employed the holographic expression of the central charge $c=3 R / 2 G_{N}$. Since the normalization of the scalar field (10) leads to the two-point function of $O$ which is proportional to the central charge $c$ (i.e. $\delta \lambda \propto \sqrt{c} \delta \phi=$ $\sqrt{c} \gamma$ ), we indeed obtain the advertised formula (2), where $\Sigma$ is given by the $\operatorname{AdS}_{2}$ slice $\rho=0$ in $\mathrm{AdS}_{3}$.

In order to study higher dimensional examples in a universal way, we would like to consider a simple holographic model, which turns out to give an excellent approximation to various explicit examples. This holographic model is obtained by approximating the exactly marginal deformation at the time slice $\tau=0$ in $\mathrm{CFT}_{d+1}$ by a $d+1$ dimensional defect brane $\Sigma$ with a tension $T$, which extends from the time slice on the AdS boundary to the bulk. This is similar to the holographic constructions in [13-15]. Note that the nature of this approximation is very similar to the hard wall model of AdS/QCD [16]. Therefore it should be regarded as a phenomenological bottom-up approach rather than a systematic and controllable approximation scheme.

In the gravity setup this prescription is simply realized by adding the defect brane action

$$
S_{\text {brane }}=T \int_{\Sigma} \sqrt{g}
$$

to the Einstein-Hilbert action. This prescription is consistent with the boundary (or defect) conformal symmetry in a way similar to the AdS/BCFT [15]. Again we can describe the perturbation (4) by the profile of a massless scalar field $\phi$. When the deformation $\delta \lambda$ is infinitesimally small, we have

$$
T \simeq n_{d} \frac{(\delta \lambda)^{2}}{R^{d+1}}
$$

where $n_{d}$ is an $O(1)$ constant which is fixed from the normalization of the two-point function (8). The Einstein equation shows that $T$ is proportional to $(\delta \lambda)^{2}$, as the bulk stress tensor is quadratic with respect to the scalar field. The dependence on $R$ can be explained by the dimensional reason or by comparing with the Janus computation. In this limit, we can treat the brane as a probe, ignoring its backreaction. Finally we impose the equation of motion with respect to the brane embedding. This requires that the action (19) is extremized and thus in the Lorentzian signature, and $\Sigma$ is the maximal area surface $\Sigma_{\max }$ that ends on the time slice $\tau=0$ at the AdS boundary. Together with (20), we reach our claim (2).

For a $\mathrm{CFT}_{d+1}$ on $R^{d+1}$, the holographic formula (2) leads to

$$
G_{\lambda \lambda}=n_{d} V_{d} \int_{\epsilon}^{\infty} \frac{d z}{z^{d+1}}=\frac{n_{d} V_{d}}{d \epsilon^{d}}
$$

which indeed agrees with (9).

Similarly we can analyze the global $\mathrm{AdS}_{d+2}$, which is described by the metric

$$
d s^{2}=R^{2}\left(-\left(r^{2}+1\right) d t^{2}+\frac{d r^{2}}{r^{2}+1}+r^{2} d \Omega_{d}^{2}\right)
$$

to obtain the information metric for a $\mathrm{CFT}_{d+1}$ on $R \times S^{d}$ :

$$
G_{\lambda \lambda}=n_{d} V_{d} \int_{0}^{r_{\infty}} \frac{r^{d}}{\sqrt{r^{2}+1}} d r=\frac{n_{d} V_{d}}{d \epsilon^{d}}+\cdots
$$

where $r_{\infty} \sim 1 / \epsilon$ is dual to the UV cutoff of the CFT. It might be curious that there appears a logarithmic divergent term $\propto \log r_{\infty}$ when $d$ is even, i.e. odd dimensional CFTs. This logarithmic term is analogous to the boundary central charge in BCFT [17]. Also it is clear from (23) that $G_{\lambda \lambda}$ is smaller than the flat space one (21) and this is due to the mass gap in CFTs on a compact space.

Another interesting example is the $d+2$ dimensional AdS Schwarzschild black hole

$$
\begin{aligned}
d s^{2} & =R^{2}\left(\frac{d z^{2}}{h(z) z^{2}}-\frac{h(z)}{z^{2}} d t^{2}+\frac{\sum_{i=1}^{d} d x_{i}^{2}}{z^{2}}\right), \\
h(z) & \equiv 1-\left(z / z_{0}\right)^{d+1},
\end{aligned}
$$


which is dual to the finite temperature CFT. The parameter $z_{0}$ is related the temperature $T$ via $z_{0}=d+1 / 4 \pi T$. The information metric is computed as

$$
\begin{aligned}
G_{\lambda \lambda} & =n_{d} V_{d} \int_{\epsilon}^{z_{0}} \frac{d z}{\sqrt{h(z)} z^{d+1}} \\
& =\frac{n_{d} V_{d}}{d}\left(\frac{1}{\epsilon^{d}}+\frac{b_{d}}{z_{0}^{d}}\right), \\
b_{d} & \equiv-1+d \int_{0}^{1} d y\left(1-y^{d+1}+\sqrt{1-y^{d+1}}\right)^{-1} \\
& =(d-1) \frac{\sqrt{\pi}}{2} \frac{\Gamma\left(1+\frac{1}{d+1}\right)}{\Gamma\left(\frac{1}{2}+\frac{1}{d+1}\right)} \geq 0 .
\end{aligned}
$$

For example, we have $b_{1}=0, b_{2} \simeq 0.70, b_{3} \simeq 1.31$.

In this thermal example (25), we are actually considering an information metric for mixed states. By generalized previous holographic analysis so that we still have the formula (2), the information metric for mixed state may be defined as follows:

$$
\frac{\operatorname{Tr}[\rho(\lambda) \rho(\lambda+d \lambda)]}{\sqrt{\operatorname{Tr}\left[\rho(\lambda)^{2}\right] \operatorname{Tr}\left[\rho(\lambda+d \lambda)^{2}\right]}}=1-2 G_{\lambda \lambda}(\delta \lambda)^{2} .
$$

However, we can describe the thermal state as a pure state in the doubled Hilbert space. In this equivalent description, our result (25) can be interpreted as (a half of) the information metric for the pure state as we will see later.

Finally we would like to study a time-dependent example in order to confirm our proposed formula (2) can be applied to such a nontrivial setup. For this purpose, we consider thermofield double (TFD) description of finite temperature state in a two-dimensional (2D) CFT:

$\left|\Psi_{\mathrm{TFD}}(t)\right\rangle \propto e^{-i\left(H^{(A)}+H^{(B)}\right) t} \sum_{n} e^{-(\beta / 4)\left(H^{(A)}+H^{(B)}\right)}|n\rangle_{A}|n\rangle_{B}$,

where $H^{(A)}$ and $H^{(B)}$ are the identical Hamiltonians for the first and second CFT of the TFD; the states $|n\rangle_{A, B}$ are the unit norm energy eigenstates in the two CFTs. This TFD setup is dual to the extended geometry of eternal AdS black hole depicted in Fig. 1.

We are interested in the inner product $\left\langle\Psi_{\mathrm{TFD}}^{\prime}(t) \mid \Psi_{\mathrm{TFD}}(t)\right\rangle$ and the information metric $G_{\lambda \lambda}$. Here, the state $\left\langle\Psi_{\mathrm{TFD}}^{\prime}(t)\right|$ is the TFD state with a Hamiltonian $H^{\prime(A)}+H^{\prime(B)}$ which is obtained by an infinitesimal exactly marginal $\lambda$ perturbation (4) with respect to each of $H^{(A)}$ and $H^{(B)}$ at the same time $t$. We argue this deformation is dual to introduce a defect brane $\Sigma$ in the Banados-Teitelboim-Zanelli (BTZ) black hole as in Fig. 1.

Let us compute $G_{\lambda \lambda}$ in a Euclidean path-integral formalism of a 2D CFT. The two-point function on $S^{1} \times R$, where $S^{1}$ is the thermal circle with periodicity $\beta$, reads

$$
\left\langle O\left(x_{1}, \tau_{1}\right) O\left(x_{2}, \tau_{2}\right)\right\rangle=\frac{\left(\frac{\pi}{\beta}\right)^{2 \Delta}}{\left[\sinh ^{2}\left(\frac{\pi\left(x_{1}-x_{2}\right)}{\beta}\right)+\sin ^{2}\left(\frac{\pi\left(\tau_{1}-\tau_{2}\right)}{\beta}\right)\right]^{\Delta}} .
$$

Then, $G_{\lambda \lambda}$ at the Euclidean time $\tau$ is expressed as

$$
\begin{aligned}
G_{\lambda \lambda}= & \frac{1}{2} \int_{-\infty}^{\infty} d x_{1} d x_{2} \int_{\beta / 4+\tau+\epsilon}^{3 \beta / 4-\tau-\epsilon} \\
& \times d \tau_{2} \int_{-\beta / 4-\tau+\epsilon}^{\beta / 4+\tau-\epsilon} d \tau_{1}\left\langle O\left(x_{1}, \tau_{1}\right) O\left(x_{2}, \tau_{2}\right)\right\rangle .
\end{aligned}
$$

Using the formula (for $0 \leq t \leq \pi$ )

$$
\begin{aligned}
& \int_{-\infty}^{\infty} d x\left(\sinh ^{2} x+\sin ^{2} t\right)^{-2} \\
& \quad=\frac{1}{\sin ^{2} t \cos ^{2} t}+(t-\pi / 2) \frac{2 \sin ^{2} t-1}{\sin ^{3} t \cos ^{3} t},
\end{aligned}
$$

we can evaluate the information metric (28) when $\Delta=2$, up to finite terms in the $\epsilon \rightarrow 0$ limit:

$$
G_{\lambda \lambda}=\frac{\pi V_{1}}{8 \epsilon}-\frac{\pi V_{1}}{2 \beta}+\frac{2 \pi^{2} V_{1}}{\beta^{2}} \tau \cot \left(\frac{4 \pi \tau}{\beta}\right)
$$

When $\tau=0$, we simply find $G_{\lambda \lambda}=\pi V_{1} / 8 \epsilon$. This is consistent with our holographic result $b_{1}=0$ in (25).

To study the real time evolution, we have only to set $\tau=i t$ and we obtain the result

$$
G_{\lambda \lambda}=\frac{\pi V_{1}}{8 \epsilon}-\frac{\pi V_{1}}{2 \beta}+\frac{2 \pi^{2} V_{1}}{\beta^{2}} \frac{\cosh \frac{4 \pi t}{\beta}}{\sinh \frac{4 \pi t}{\beta}} t
$$

At late time $t \gg \beta$, it grows linearly:

$$
G_{\lambda \lambda} \simeq \frac{\pi V_{1}}{8 \epsilon}+\frac{2 \pi^{2} V_{1}}{\beta^{2}} t
$$

We now turn to the holographic computation in the BTZ black hole, where the region (I) in Fig. 1 is described by the metric (we set $\beta=2 \pi$ for simplicity)

$$
d s^{2}=R^{2}\left(-\sinh ^{2} \rho d t^{2}+d \rho^{2}+\cosh ^{2} \rho d x^{2}\right) .
$$

We can continue into the region (II) by setting $\kappa=-i \rho$ and $\tilde{t}=t+\pi i / 2$. In the region (II), if we specify $\Sigma$ by $\kappa=\kappa(\tilde{t})$ of $\Sigma$, its volume is given by

$$
\operatorname{Vol}(\Sigma)=R^{2} V_{1} \int d \tilde{t} \cos \kappa \sqrt{\sin ^{2} \kappa-(\partial \kappa / \partial \tilde{t})^{2}} .
$$

We define $\kappa_{*}\left(0 \leq \kappa_{*}<\pi / 4\right)$ to be the value of $\kappa$ where $\partial \kappa / \partial \tilde{t}=0$. We can maximize the volume (34) and extend the solution into the region (I) in a way similar to [18]. In the end we obtain the following expression of $\operatorname{Vol}\left(\Sigma_{\max }\right)$ as a function of $t$ in terms of the parameter $\kappa_{*}$ : 


$$
\begin{aligned}
\frac{\operatorname{Vol}(\Sigma)}{R^{d+1} V_{1}} & =2 \sinh \rho_{\infty}+2 \int_{0}^{\kappa_{*}} d \kappa \frac{\cos \kappa}{\sqrt{\sin ^{2}\left(2 \kappa_{*}\right) / \sin ^{2}(2 \kappa)-1}}-2 \int_{0}^{\rho_{\infty}} d \rho \frac{\cosh \rho\left[\sqrt{\sinh ^{2}(2 \rho)+\sin ^{2}\left(2 \kappa_{*}\right)}-\sinh (2 \rho)\right]}{\sqrt{\sinh ^{2}(2 \rho)+\sin ^{2}\left(2 \kappa_{*}\right)}}, \\
t & =\int_{0}^{\kappa_{*}} \frac{d \kappa}{\sin \kappa \sqrt{1-\sin ^{2}(2 \kappa) / \sin ^{2}\left(2 \kappa^{*}\right)}}-\int_{0}^{\rho_{\infty}} \frac{d \rho}{\sinh \rho \sqrt{1+\sinh ^{2}(2 \rho) / \sin ^{2}\left(2 \kappa^{*}\right)}},
\end{aligned}
$$

where $\rho_{\infty}$ is the UV cutoff such that $e^{\rho_{\infty}} \propto 1 / \epsilon$. In the late time limit (i.e. $\kappa_{*} \rightarrow \pi / 4$ ) we find the finite part $\operatorname{Vol}_{\text {finite }}(\Sigma) / R^{d+1}$ approaches to $V_{1} t$, which agrees with $2 G_{\lambda \lambda}$ in (32). This linear $t$ growth clearly comes from the Einstein-Rosen bridge as already noted in [6]. In Fig. 2 we plotted the holographic result versus the CFT result, which shows only a very small deviation. For example, in the limit $t \rightarrow 0\left(\right.$ or $\left.\kappa_{*} \rightarrow 0\right)$ we find $\operatorname{Vol}_{\text {finite }}(\Sigma) /\left(R^{d+1} V_{1}\right) \simeq$ $(2 / \pi) t^{2}$, while $2 G_{\lambda \lambda} / V_{1} \simeq \frac{2}{3} t^{2}$.

In this Letter, we introduced an information metric in CFTs and proposed its holographic formula based on a simple probe model. We presented nontrivial evidence which supports our proposal. It might be interesting to note that if we normalize the two-point function such that it is proportional to a central charge or if we employ some combination of energy stress tensors (related to some metric perturbations), we have the estimation $G_{\lambda \lambda} \sim\left[\operatorname{Vol}\left(\Sigma_{\max }\right) / G_{N} R\right]$, which is the same formula argued in [6] to measure the amount of complexity.

We are very grateful to Adam Brown and Leonard Susskind for careful reading of the draft of this paper and giving us valuable comments. We also thank David Berenstein, Andreas Karch, Shunji Matsuura, Masahiro Nozaki, Shinsei Ryu, Kostas Skenderis, Yu Watanabe, Toby Wiseman, and Beni Yoshida for useful comments. We are also thankful to the organizers and participants of the workshop "Numerical approaches to the holographic principle, quantum gravity and cosmology," held in YITP, Kyoto University, where the results in this paper have been presented. T. T. is grateful to the organizers and participants

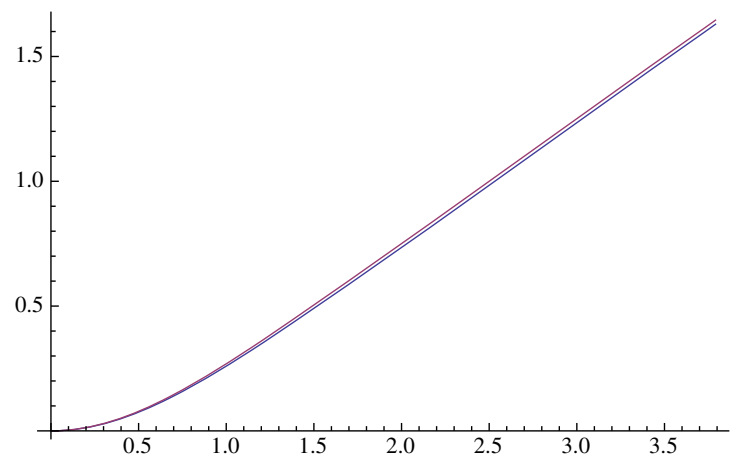

FIG. 2 (color online). We compare the finite part of our holographic result $\operatorname{Vol}(\Sigma) /\left(R^{d+1} V_{1}\right)$ (blue) with that of our CFT result $2 G_{\lambda \lambda} / V_{1}$ (red) as a function of the time $t$ for the TFD state. They deviate only slightly. We set $\beta=2 \pi$. of the KITP program "Entanglement in Strongly Correlated Quantum Matter," held in KITP, UCSB, where important progresses in this paper have been made. T. N, N. S., and $\mathrm{K}$. W. are supported by JSPS. T. T. is supported by JSPS Grant-in-Aid for Scientific Research (B) No. 25287058. T. $\mathrm{T}$. is also supported by World Premier International Research Center Initiative (WPI Initiative) from the Japan Ministry of Education, Culture, Sports, Science and Technology (MEXT).

[1] A. Strominger and C. Vafa, Phys. Lett. B 379, 99 (1996).

[2] J. M. Maldacena, Adv. Theor. Math. Phys. 2, 231 (1998); Int. J. Theor. Phys. 38, 1113 (1999).

[3] G. 't Hooft, arXiv:gr-qc/9310026; L. Susskind, J. Math. Phys. (N.Y.) 36, 6377 (1995); D. Bigatti and L. Susskind, arXiv:hep-th/0002044.

[4] J. M. Maldacena, J. High Energy Phys. 04 (2003) 021.

[5] S. Ryu and T. Takayanagi, Phys. Rev. Lett. 96, 181602 (2006); J. High Energy Phys. 08 (2006) 045; V. E. Hubeny, M. Rangamani, and T. Takayanagi, J. High Energy Phys. 07 (2007) 062.

[6] L. Susskind, arXiv:1403.5695; arXiv:1411.0690.

[7] D. Stanford and L. Susskind, Phys. Rev. D 90, 126007 (2014).

[8] S. L. Braunstein and C. M. Caves, Phys. Rev. Lett. 72, 3439 (1994).

[9] S-J. Gu, Int. J. Mod. Phys. B 24, 4371 (2010).

[10] M. Nozaki, S. Ryu, and T. Takayanagi, J. High Energy Phys. 10 (2012) 193; M. Miyaji and T. Takayanagi, Prog. Theor. Exp. Phys. 2015, 073 B03 (2015); M. Miyaji, T. Numasawa, N. Shiba, T. Takayanagi, and K. Watanabe, Phys. Rev. Lett. 115, 171602 (2015).

[11] D. Bak, M. Gutperle, and S. Hirano, J. High Energy Phys. 05 (2003) 072.

[12] D. Bak, M. Gutperle, and S. Hirano, J. High Energy Phys. 02 (2007) 068.

[13] A. Karch and L. Randall, J. High Energy Phys. 05 (2001) 008; 06 (2001) 063.

[14] T. Azeyanagi, A. Karch, T. Takayanagi, and E. G. Thompson, J. High Energy Phys. 03 (2008) 054.

[15] T. Takayanagi, Phys. Rev. Lett. 107, 101602 (2011); M. Fujita, T. Takayanagi, and E. Tonni, J. High Energy Phys. 11 (2011) 043.

[16] J. Erlich, E. Katz, D. T. Son, and M. A. Stephanov, Phys. Rev. Lett. 95, 261602 (2005).

[17] M. Nozaki, T. Takayanagi, and T. Ugajin, J. High Energy Phys. 06 (2012) 066.

[18] T. Hartman and J. Maldacena, J. High Energy Phys. 05 (2013) 014. 